\title{
Diagnosis of infection in patients undergoing extracorporeal membrane oxygenation: A case-control study
}

\author{
Marina Pieri, MD, ${ }^{\mathrm{a}}$ Teresa Greco, MSc, ${ }^{\mathrm{a}}$ Michele De Bonis, MD, ${ }^{\mathrm{b}}$ Giulia Maj, MD, ${ }^{\mathrm{a}}$ Luca Fumagalli, MD, ${ }^{\mathrm{c}}$ \\ Alberto Zangrillo, $\mathrm{MD},{ }^{\mathrm{a}}$ and Federico Pappalardo, $\mathrm{MD}^{\mathrm{a}}$
}

Objective: Diagnosis of infection in patients receiving extracorporeal membrane oxygenation is challenging in clinical practice but represents a crucial aspect of the upgrading of therapeutic options. The aim of this study was to analyze the role of C-reactive protein and procalcitonin in the diagnosis of infection in patients requiring extracorporeal membrane oxygenation and to assess the difference between venovenous and venoarterial extracorporeal membrane oxygenation settings.

Methods: A case-control study was performed on 27 patients. Serum values of procalcitonin and C-reactive protein were analyzed according to the presence of infection.

Results: Forty-eight percent of patients had infection. Gram-negative bacteria were the predominant pathogens, and Candida albicans was the most frequent isolated microorganism. Procalcitonin had an area under the curve of $0.681(P=.0062)$ for the diagnosis of infection in the venoarterial extracorporeal membrane oxygenation group but failed to discriminate infection in the venovenous extracorporeal membrane oxygenation group $(P=.14)$. The area under the curve of $C$-reactive protein was $0.707(P<.001)$ in all patients receiving extracorporeal membrane oxygenation. In patients receiving venoarterial extracorporeal membrane oxygenation, procalcitonin had good accuracy with $1.89 \mathrm{ng} / \mathrm{mL}$ as the cutoff (sensitivity $=87.8 \%$, specificity $=50 \%$ ) and C-reactive protein with $97.70 \mathrm{mg} / \mathrm{L}$ as the cutoff (sensitivity $=85.3 \%$, specificity $=41.6 \%$ ). The procalcitonin and C-reactive protein combined assay had a sensitivity of $87.2 \%$ and specificity of $25.9 \%$. Four variables were identified as statistically significant predictors of infection: procalcitonin and $\mathrm{C}$-reactive protein combined assay (odds ratio, 1.184; $P<.001$ ), age (odds ratio, $0.980 ; P<.001$ ), presence of infection before extracorporeal membrane oxygenation implantation (odds ratio, 1.782; $P<.001$ ), and duration of extracorporeal membrane oxygenation support (odds ratio, 1.056; $P<.001)$.

Conclusions: Traditional and emerging inflammatory biomarkers, especially if compounded in the procalcitonin and C-reactive protein combined assay, can aid in the diagnosis of infection in patients undergoing venoarterial extracorporeal membrane oxygenation. (J Thorac Cardiovasc Surg 2012;143:1411-6)

\section{Supplemental material is available online.}

Extracorporeal membrane oxygenation (ECMO) represents an effective tool for the treatment of heart and lung failure. Nevertheless, clinicians may encounter many hardships in the management of this innovative therapeutic

\footnotetext{
From the Department of Cardiothoracic and Vascular Anesthesia and Intensive Care, ${ }^{\mathrm{a}}$ Department of Cardiac Surgery, ${ }^{\mathrm{b}}$ Istituto Scientifico San Raffaele, Milan, Italy; and Department of Infectious Diseases, ${ }^{\mathrm{c}}$ Istituto Scientifico San Raffaele Turro, Milan, Italy.

Funding: The study was entirely supported by departmental funds.

Disclosures: Authors have nothing to disclose with regard to commercial support.

Received for publication Sept 2, 2011; revisions received Dec 16, 2011; accepted for publication Jan 4, 2012; available ahead of print Feb 1, 2012.

Address for reprints: Federico Pappalardo, MD, Department of Cardiothoracic and Vascular Anesthesia and Intensive Care, Istituto Scientifico San Raffaele, Via Olgettina 60 Milan, 20132 Italy (E-mail: pappalardo.federico@hsr.it). $0022-5223 / \$ 36.00$

Copyright (c) 2012 by The American Association for Thoracic Surgery doi:10.1016/j.jtcvs.2012.01.005
}

option. One of the most problematic challenges is to define the presence and onset of infection and sepsis, because these critically ill patients invariably present clinical signs of systemic inflammatory response. ${ }^{1}$ In such patients, the systemic inflammatory response syndrome (SIRS) is due to both the underlying acute disease and the host response to the presence of the extracorporeal circuit itself. The issue is critical because mortality in sepsis is high and early therapeutic intervention can improve prognosis. ${ }^{2}$ On the other hand, broad use of antibiotics in all patients with SIRS may lead to a prevalence of resistant strains with increasing toxicity and costs. Moreover, when ECMO is used as a bridge to other potential therapeutic options, including organ transplant and long-term assist device implantation, the presence of infection is to be rigorously established to evaluate whether patients' eligibility criteria are met.

This study analyzes the role of C-reactive protein (CRP) and procalcitonin (PCT) in the diagnosis of bacterial and fungal infection in critically ill patients requiring ECMO, and a new diagnostic test for clinical practice is presented. 


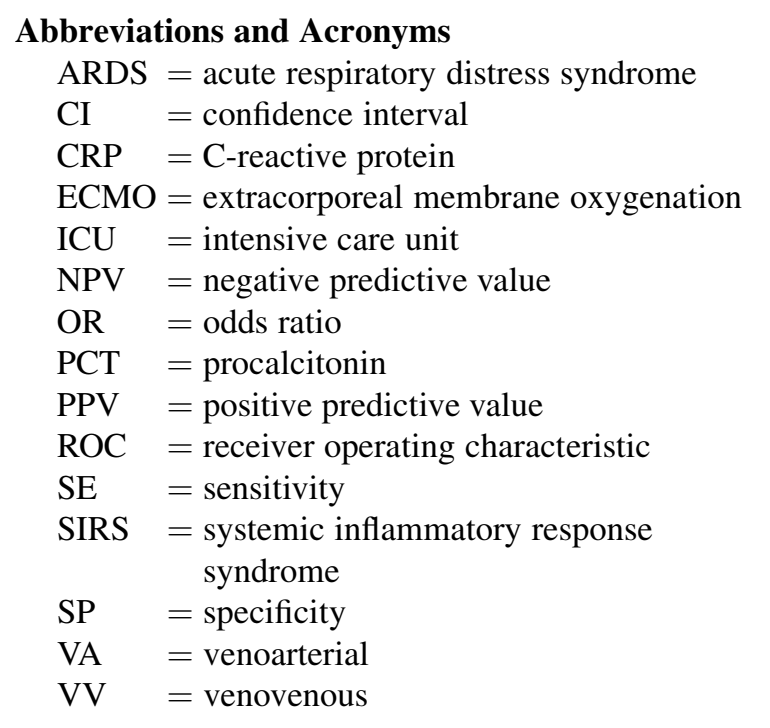

\section{MATERIALS AND METHODS}

\section{Setting and Study Population}

After ethical committee approval, we carried out an observational study on 27 adult patients who were treated with venovenous (VV) ECMO or venoarterial (VA) ECMO between November 2009 and January 2011, and admitted to the cardiovascular intensive care unit (ICU) of Istituto Scientifico San Raffaele.

Eleven patients with severe acute respiratory distress syndrome (ARDS) were treated with VV ECMO, and 16 patients with refractory cardiogenic shock were treated with VA ECMO. Among patients receiving VV ECMO, 7 had confirmed or strongly suspected ARDS due to H1N1 influenza A infection. The other 4 patients treated with VV ECMO had ARDS after polytrauma, cardiogenic shock, pneumonia with negative microbiological tests, and pancreatic surgery. None of the patients receiving VA ECMO had evidence of infection at the time of cannulation, except 1 patient with H1N1 myocarditis with positive pharyngeal swab. Thirteen of 27 patients had infection, and 14 patients were part of the control group.

\section{Clinical Management}

The ECMO circuit setup consisted of a centrifugal pump and a heparincoated polymethylpentene oxygenator. Two patients received a circuit without an oxygenator for isolated refractory acute heart failure with preserved pulmonary function. Because all the other components of the circuit and clinical management were identical to those of the patients receiving VA ECMO, these 2 patients were included in the VA ECMO group.

All patients were intubated during ECMO support, except 1 patient with non-H1N1-related pneumonia who received VV ECMO after failure of noninvasive ventilation. Baseline monitoring consisted of invasive pressure monitoring, pulse oximetry, continuous electrocardiography, urine output, and central venous pressure. Chest radiogram and transesophageal echocardiographic evaluations were performed and repeated as needed. Thermodilution cardiac output measurements were done. Arterial blood gas analysis was performed every hour. Evaluation of hematocrit, white blood cell count, coagulative profile (activated clotting time, prothrombin time, partial thromboplastin time, antithrombin, free hemoglobin, fibrinogen, D-dimer, and C protein), and electrolytes were performed every 6 hours or when deemed necessary. Continuous clinical monitoring for bleeding was performed, and laboratory measurement was requested more frequently if necessary. Daily evaluation of cardiac enzymes, hepatic enzymes, and creatinine was performed. Blood gas analysis from radial artery, pre-oxygenator blood, and post-oxygenator blood was performed daily. Continuous evaluation of the ECMO circuit, clotting formation, and pressure difference was performed.

Hemolysis was monitored by daily measurement of serum concentrations of lactate dehydrogenase and free hemoglobin. Hemolysis was diagnosed in the presence of lactate dehydrogenase serum levels greater than $1000 \mathrm{U} / \mathrm{L}$ and a serum free hemoglobin level greater than $40 \mathrm{mg} / \mathrm{L}$ in 2 consecutive samples within 24 hours. In addition to these elements, a reduction of hemoglobin serum values and eventually hematuria strongly supported the diagnosis.

Anticoagulation was achieved by intravenous continuous infusion of unfractionated heparin titrated to maintain an activated partial thromboplastin time between 45 and 50 seconds. In case of heparin-induced thrombocytopenia, unfractionated heparin was discontinued and intravenous bivalirudin infusion was started.

In addition to anticoagulation therapy, standard intravenous therapy included gastric prophylaxis with histamine H2-receptor antagonists, antibiotic prophylaxis with cefazolin for the first 48 hours after cannulation procedure, hydration, and diuretics. Patients with defined or suspected H1N1 influenza A infection received antiviral therapy with oseltamivir $150 \mathrm{mg}$ twice per day and broad-spectrum antibiotics.

\section{Infection Monitoring and Diagnosis}

For infection surveillance and management, we followed international guideline recommendations. ${ }^{3}$ Infection diagnosis followed Centers for Disease Control and Prevention criteria for specific types of infections in the acute care setting. The Centers for Disease Control and Prevention developed criteria for the diagnosis of 48 specific sites or types of infection and grouped them into 13 major site categories. The diagnosis is based on the presence of a combination of criteria (including clinical signs and symptoms, vital signs, microbiological investigations, and clinical evidence) that are different and clearly stated according to the different types of infection. ${ }^{4}$

Three sets of blood cultures, deep tracheal aspirate or bronchoalveolar lavage, and urine samples were sent periodically to the laboratory for microbiological examination. Whenever an intravascular device was changed or removed, the tip was sent for microbiological investigation. Three sets of blood cultures were obtained in case of fever $\left(38^{\circ} \mathrm{C}\right)$ or increase in white blood cell count. A deep tracheal aspirate and eventually a bronchoalveolar lavage were obtained whenever respiratory infection was suspected, such as in changes on chest radiography or evidence of worsening in respiratory performance.

A urinary sample was sent for cultural examination whenever standard monitoring cultural examinations were performed and whenever deemed appropriate. Because the ECMO circuit included the heat exchanger, the changing of temperature alone was not regarded as a sign of infection. When infection was clinically suspected, an empiric wide-spectrum antibiotic therapy was administered immediately after collecting samples for microbiology. Once the causative pathogen was known and the antibiogram was available, the antibiotic therapy was pathogen-tailored. The oxygenator and cannulae were not changed after infection identification.

\section{Parameters of Interest}

Two separate blood samples were collected each morning to determine CRP and PCT values, and only 1 value of CRP and of PCT was analyzed per day. PCT values were detected with the Elecsys BRAHMS PCT assay (Brahms, Berlin, Germany), and CRP was determined by a turbidimetric immunoassay (Siemens Healthcare Diagnostics Inc, Deerfield, Ill).

\section{Statistical Analysis}

Analysis of the parameters of interest (CRP and PCT) was performed on the values measured once daily during the ECMO run and not on baseline values. Categoric variables are expressed as numbers and percentages, 
TABLE 1. Study population baseline characteristics

\begin{tabular}{|c|c|c|c|c|}
\hline Par: & $\begin{array}{c}\text { Total } \\
(n=27)\end{array}$ & $\frac{\text { VV ECMO }}{(n=11)}$ & $\frac{\text { VA ECMO }}{(n=16)}$ & $\begin{array}{c}P \\
\text { value }\end{array}$ \\
\hline ge, y & $54.9 \pm 11.3$ & $49.6 \pm 13.1$ & $58.6 \pm 8.6$ & .01 \\
\hline ender (male), $n$ & $20(74.1 \%)$ & $6(54.6 \%)$ & $14(87.5 \%)$ & .07 \\
\hline Weight, kg & $73.2 \pm 13.6$ & $73.1 \pm 17.2$ & $73.3 \pm 11.1$ & .7 \\
\hline Height, cm & $170.2 \pm 6.8$ & $168.5 \pm 9.3$ & $171.2 \pm 43.5$ & .5 \\
\hline MI & $25.5 \pm 3.4$ & $26.3 \pm$ & $25.0 \pm$ & .3 \\
\hline BSA & $1.9 \pm 0.2$ & $1.9 \pm 0.2$ & $1.9 \pm 0.2$ & .9 \\
\hline $\begin{array}{l}\text { Medical } \\
\text { hospitalization, } \mathrm{n}\end{array}$ & $13(48.2 \%)$ & $7(63.6 \%)$ & $6(37.5 \%)$ & .8 \\
\hline $\begin{array}{l}\text { Elective surgery } \\
\text { hospitalization, } \mathrm{n}\end{array}$ & $12(44.4 \%)$ & $3(27.3 \%)$ & $9(56.3 \%)$ & .0 \\
\hline $\begin{array}{r}\text { Nonelective surgery } \\
\text { hospitalization, } n\end{array}$ & $2(7.4 \%)$ & $1(9.1 \%)$ & $1(6.3 \%)$ & .9 \\
\hline APACHE II score & $14.9 \pm 7.1$ & $12.1 \pm 4.6$ & $16.8 \pm 8.0$ & .1 \\
\hline SOFA score & $8.0 \pm 3.3$ & $7.8 \pm 4.0$ & $8.2 \pm 2.8$ & .3 \\
\hline
\end{tabular}

ECMO, Extracorporeal membrane oxygenation; $B M I$, body mass index; $B S A$, body surface area; APACHE II, Acute Physiology And Chronic Health Evaluation II; SOFA, Sequential Organ Failure Assessment.

whereas continuous variables are shown as mean \pm standard deviation or as median and interquartile range if the standard deviation is larger than the mean in magnitude.

For group comparisons, binomial test was used for dichotomous variables, whereas Mann-Whitney $U$ test or Mood's median test was performed for assay mean or median difference, respectively. Moreover, the Spearman rank-order correlation coefficient was used to compare PCT and CRP variation.

The relation among PCT, CRP, infection, and other parameters under study was analyzed with a generalized estimating equations model, taking into account correlations within patients' measurements, assuming the same correlation between any 2 elements of a cluster (exchangeable correlation matrix). ${ }^{5,6}$

Receiver operating characteristic (ROC) analysis ${ }^{7}$ was used to identify the most appropriate cutoff values for the diagnosis of infection. The performance of the diagnostic tests was evaluated with the nonparametric method of DeLong and colleagues, ${ }^{8}$ assessing the difference from the nondiscriminating ROC curve (area under the curve $=0.5$ ). Sensitivity (SE), specificity (SP), positive predictive value (PPV), negative predictive value (NPV), and misclassification rate were assessed with reference to PCT and
CRP cutoff values, and their $95 \% \mathrm{CI}$ was calculated with binomial approximation. Calculation of SE, SP, PPV, and NPV is referred to in the text as "accuracy evaluation." To set the false-negative count to zero and to confirm a negative test result, both tests were assessed in parallel with "the OR rule" (positive diagnosis if either test is positive and negative diagnosis if both tests are negative). ${ }^{9}$ This resulted in the creation of a new variable that we called the "PCT and CRP combined assay."

Because of the presence of a conditional dependence between the 2 diagnostic tests, we calculated the covariance of SE and SP and corrected the estimate of the diagnostic performance accordingly. ${ }^{10}$ Data were analyzed with SAS 2002-2008 software (release 9.2 by SAS Institute Inc, Cary, NC).

\section{RESULTS}

The study population baseline characteristics are shown in Table 1 , and the study population data are shown in Table 2. The mean number of days on ECMO support was $6.6 \pm 3.8$, specifically $7.0 \pm 2.7$ days in $\mathrm{VV}$ cases and $6.4 \pm 4.5$ days in VA cases.

Eight patients (29.6\%) died on ECMO, and no significant difference in mortality between the VV ECMO and VA ECMO groups was observed. The median ICU stay was 15 (7-32) days, and the median hospital stay was $16(10$ 51) days, with no statistically significant difference between the groups.

No patient had hemolysis. Thirteen patients $(48 \%)$ had bacterial or fungal infection: In 7 patients $(26 \%)$, infection was acquired while on extracorporeal support, and the other 6 patients $(22 \%)$ had preexisting infection. An overview of infections is shown in Table 3.

We observed 3 episodes of bloodstream infection and 10 infections with negative blood cultures. Among patients without bloodstream infection, we reported 8 pneumonias (7 episodes with positive tracheal specimen and 1 case with positive bronchoalveolar lavage) and 2 episodes of infection with negative microbiological investigations.

Twelve different pathogens were isolated in a total of 13 isolates: gram-negative bacteria were the most common $(54 \%)$, followed by fungi $(23 \%)$ and gram-positive

TABLE 2. Study population data

\begin{tabular}{|c|c|c|c|c|}
\hline Parameter & Total $(n=27)$ & VV ECMO $(n=11)$ & $\operatorname{VA~} \operatorname{ECMO}(n=16)$ & $P$ value \\
\hline $\mathrm{CRP}, \mathrm{mg} / \mathrm{L}$ & $200.7(78.4-240.9)$ & $216.9(142.3-264.0)$ & $111.3(76.0-206.4)$ & .03 \\
\hline $\mathrm{CRP}$ maximum value registered, $\mathrm{d}$ & $2(1-4)$ & $2(1-3)$ & $3(1-4)$ & .6 \\
\hline $\mathrm{PCT}, \mathrm{ng} / \mathrm{mL}$ & $3.5(0.6-15.1)$ & $4.2(0.3-13.4)$ & $3.1(0.9-21.0)$ & .9 \\
\hline PCT maximum value registered, $\mathrm{d}$ & $2(1-4)$ & $2(0-5)$ & $1.5(1-3.5)$ & .2 \\
\hline $\mathrm{WBC}, 10^{9} / \mathrm{L}$ & $14.2(9.9-17.9)$ & $11.5(5.3-17.4)$ & $14,6(11.4-17.9)$ & .2 \\
\hline WBC maximum value registered, $\mathrm{d}$ & $3(1-6)$ & $4(3-7)$ & $2(0.5-3.5)$ & .1 \\
\hline $\mathrm{PC}, \%$ & $48.6(42.1-72.3)$ & $47.9(47.0-64.7)$ & $48.6(42.1-72.3)$ & .9 \\
\hline PC maximum value registered, $\mathrm{d}$ & $2(1-6)$ & $5(2-7)$ & $2(0.5-5)$ & 6 \\
\hline Lactates, $\mathrm{mmol} / \mathrm{L}$ & $4.22(2.36-6.73)$ & $2.66(1.9-7.8)$ & $4.5(2.9-6.1)$ & .5 \\
\hline ECMO, d & $6.6 \pm 3.8$ & $7.0 \pm 2.7$ & $6.4 \pm 4.5$ & .4 \\
\hline Hospital stay, d & $16(10-51)$ & $17(11-41)$ & $15.5(7-52)$ & .06 \\
\hline ICU stay, d & $15(7-32)$ & $15(10-32)$ & $15.5(6-29.5)$ & .07 \\
\hline Death on ECMO, $n$ & $8(29.6 \%)$ & $4(36.4 \%)$ & $4(25.0 \%)$ & .9 \\
\hline Infection, $\mathrm{n}$ & $13(48.1 \%)$ & $5(45.5 \%)$ & $8(50 \%)$ & .8 \\
\hline
\end{tabular}

$E C M O$, Extracorporeal membrane oxygenation; $C R P$, C-reactive protein; $P C T$, procalcitonin; $W B C$, white blood cell; $P C$, protein $\mathrm{C} ; I C U$, intensive care unit. 
TABLE 3. Infection overview

\begin{tabular}{|c|c|c|c|}
\hline Patient & $\begin{array}{c}\text { Extracorporeal } \\
\text { support }\end{array}$ & $\begin{array}{c}\text { Type of } \\
\text { infection* }\end{array}$ & Pathogen \\
\hline 1 & VV ECMO & Pneumonia & Acinetobacter baumannit \\
\hline \multirow[t]{2}{*}{2} & VV ECMO & Bloodstream & Candida albicans \\
\hline & & Bloodstream & $\begin{array}{l}\text { Corynebacterium } \\
\text { minutissimum }\end{array}$ \\
\hline 3 & VV ECMO & Pneumonia & $\begin{array}{l}\text { Stenotrophomonas } \\
\text { maltophilia }\end{array}$ \\
\hline 4 & VV ECMO & Pneumonia & Enterobacter aerogenes \\
\hline 5 & VV ECMO & Pneumonia & No pathogen isolated \\
\hline 6 & VA ECMO & Pneumonia & Serratia marcescens \\
\hline 7 & VA ECMO & Pneumonia & Aspergillus fumigatus \\
\hline \multirow[t]{2}{*}{8} & VA ECMO & $\begin{array}{l}\text { Pneumonia }+ \\
\text { bloodstream }\end{array}$ & Escherichia coli \\
\hline & & Pneumonia & Klebsiella pneumoniae \\
\hline 9 & VA ECMO & Bloodstream & Candida albicans \\
\hline 10 & VA ECMO & Pneumonia & MRSA \\
\hline 11 & VA ECMO & Pneumonia & Haemophilus influenzae \\
\hline 12 & VA ECMO & Cholecystitis & No pathogen isolated \\
\hline 13 & VA ECMO & Pneumonia & Staphylococcus aureus \\
\hline
\end{tabular}

microorganisms $(23 \%)$. The most frequently isolated pathogen was a fungus: Candida albicans was isolated in $2 \mathrm{ep}-$ isodes. Moreover, Staphylococcus aureus was the most frequent isolated gram-positive bacterium $(67 \%)$.

PCT mean values were associated with the ECMO configuration (regression coefficient $=-9.327, P=.047$ ), adjusted for CRP value (regression coefficient $=0.042$, $P=.03)$. Therefore, a stratified analysis according to the ECMO configuration was mandatory to detect the optimal cutoff for the diagnosis of infection. On the contrary, we found that CRP mean values were significantly associated with age (regression coefficient $=-5.075, P=.002$ ) and gender (regression coefficient $=-90.752, P=.01$ ) but not with the ECMO configuration. Thus, a single cutoff value for CRP was appropriate for both the VV ECMO and VA ECMO groups. In addition, we found a significant positive correlation between PCT and CRP (Spearman's rank correlation coefficient $=0.38, P<.001$ ).

The ROC curve of PCT was not significantly different from the nondiscriminating ROC curve $(P=.07)$. However, if we distinguish between the 2 types of ECMO configuration, we found that PCT is a reliable biomarker for the diagnosis of infection in patients undergoing VA ECMO (area under the curve, $0.681 ; 95 \% \mathrm{CI}, 0.551-0.811 ; P=.0062$ ) (Figure E1) but not in patients receiving VV ECMO $(P=.14)$. On the other hand, the ROC analysis confirmed a good accuracy of CRP in the discrimination of patients with and without infection in all ECMO cases (area under the curve, 0.707; 95\% CI, 0.623-0.791; $P<.001)$ (Figure E2).
In the light of these data, we focused only on patients receiving VA ECMO. We performed an accuracy evaluation of PCT as a possible diagnostic test for infection and compared it with CRP. We confirmed a good accuracy of the PCT diagnostic test with $1.89 \mathrm{ng} / \mathrm{mL}$ as the optimal cutoff point $(\mathrm{SE}=87.8 \%$ [95\% CI, 78.6-96.9], $\mathrm{SP}=50 \%$ [95\% CI, 33.7-66.3], PPV $=70.5 \%$ [95\% CI, 59-81.9], $\mathrm{NPV}=75 \% \quad[95 \% \mathrm{CI}, 57.7-92.3]$, misclassification rate $=28.2 \%[95 \%$ CI, 18.7-37.8]) and the CRP diagnostic test with $97.70 \mathrm{mg} / \mathrm{L}$ as the cutoff $(\mathrm{SE}=85.3 \%[95 \% \mathrm{CI}$, 76.9-93.7], $\mathrm{SP}=41.6 \% \quad[95 \% \quad \mathrm{CI}, \quad 30.6-52.6]$, $\mathrm{PPV}=56.3 \%[95 \% \mathrm{CI}, 46.7-65.9], \mathrm{NPV}=76.2 \%$ [95\% CI, 63.3-89.1], misclassification rate $=37.9 \%$ [95\% CI, 30-45.8]).

The probability of a negative test result in infection detection, when performing both diagnostic tests in parallel, decreased to $0.018(\mathrm{SE}=98.2 \%, \mathrm{SP}=20.8 \%)$. Because of the presence of a conditional dependence between PCT and CRP, however, the estimate of the diagnostic performance of the 2 tests in parallel was adjusted according to the SE and SP covariance of 0.110 and 0.051 , respectively $(\mathrm{SE}=87.2 \%, \mathrm{SP}=25.9 \%)$.

Four variables were identified as statistically significant predictors of infection: parallel test result, that is, the "PCT and CRP combined assay" (odds ratio [OR] = $1.184 ; 95 \%$ confidence interval [CI], 1.073-1.307, $P<.001)$, age $(\mathrm{OR}=0.980 ; 95 \%$ CI, 0.972-0.988, $P<.001$ ), presence of infection before ECMO implantation $(\mathrm{OR}=1.782 ; 95 \% \mathrm{CI}, 1.462-2.171, P<.001)$, and duration of ECMO support (OR $=1.056$; 95\% CI, 1.029-1.084, $P<.001)$.

\section{DISCUSSION}

This is the first study to address the role of traditional and emerging biomarkers in the diagnosis of infection in patients undergoing ECMO. The most interesting result is the creation of a new test, the "PCT and CRP combined assay," which was proved to be a statistically significant predictor of infection in patients receiving VA ECMO, along with younger age, presence of infection before the ECMO support, and longer duration of the ECMO support. It is a promising finding, especially because infection in patients undergoing extracorporeal support is a major complication.

Infection rates and causal organisms of 20,741 patients receiving ECMO were recently reported in a query conducted on the Extracorporeal Life Support Organization registry on behalf of the Extracorporeal Life Support Organization Task Force on Infections and ECMO.${ }^{11}$ Our study confirmed similar results, because we observed that bacterial or fungal infection developed in approximately half of the patients receiving ECMO. Of note, such infections were not always correlated to the cannulation site; rather, we observed mainly bloodstream infections and pneumonia. 
A recent study by Sun and colleagues ${ }^{12}$ reported an ECMO-related infection rate of $13.5 \%$ in a series of 334 patients. Because the aim of our study was to establish the role of serum biomarkers in the diagnosis of infection in patients on ECMO, we studied the day-by-day changes in biomarker values in patients with or without infection during ECMO support regardless of whether the infection was preexisting. Among the 13 patients with infection of our study, only 7 $(26 \%)$ had ECMO-related infection. Furthermore, Bizzarro and colleagues ${ }^{11}$ reported an infection rate of $20.9 \%$ in 2298 adult patients, which is similar to the infection rate we observed in our sample.

However, few patients on ECMO are 100\% infection free. Even at the time of cannulation, it is difficult to define whether a patient is truly infection free, because ECMO is often used in patients with ARDS or who have undergone elective or nonelective surgery.

Moreover, ECMO is frequently used as a bridge to other therapies, such as heart or lung transplantation and longterm ventricular assist device implantation. However, the presence of infection jeopardizes patients' candidacy to receive these therapeutic options. For this reason, reliable tools for the diagnosis of infection are strongly needed, irrespective of the speculations concerning whether the infection can be defined as related to ECMO or not.

Traditional inflammatory marker assays (eg, CRP and white blood cell count) are often distorted in such patients by the presence of non-septic SIRS. Because the extracorporeal circuit is a foreign body in constant contact with the patient's blood, it triggers the activation of defense mechanisms, resulting in a marked inflammatory response.

In regard to PCT, patients with established sepsis diagnosis generally have higher serum levels than those with SIRS only, and patients with severe sepsis and septic shock are reported to have the highest levels. ${ }^{13,14}$ However, no study previously analyzed PCT as an infection marker in patients undergoing extracorporeal support. Few pioneering studies described PCT kinetics in cardiopulmonary bypass, which is routinely used in cardiac surgery.

Significantly increased PCT values are not observed in the absence of postoperative complications, ${ }^{15}$ and PCT levels in patients with infection are generally higher than in patients with noninfectious complications. ${ }^{16}$ Nevertheless, it is difficult to recommend cutoff points.

The presence of H1N1 influenza A pneumonia in 7 of the 27 patients is not a limitation of the study, because PCT is supposed to have low or undetectable levels in the case of viral infection. ${ }^{17-19}$ Furthermore, some studies also successfully demonstrated that PCT can assist in bacterial infection diagnosis and tailored antibiotic use in patients with H1N1 pneumonia. ${ }^{20,21}$

In regard to the other acute-phase reactants, a record of increased PCT values must be interpreted cautiously. There is an overlap between SIRS and sepsis, and many situations other than infection may trigger PCT synthesis and release, including inhalational injury, burn injury, pancreatitis, mechanical trauma, extensive surgery (eg, aortic, cardiac, colonic), ${ }^{16,22,23}$ and cardiogenic shock. ${ }^{24}$ We acknowledge that many of these conditions were present in our study population and may have partly influenced the PCT values and our results. Of note, several studies available in the literature concluded that a combination of PCT and CRP would provide the most useful information in the diagnosis of infection. $^{21,22}$

Our study demonstrated that a concomitant PCT and CRP assay is useful in the critical care setting of patients undergoing VA ECMO, because PCT and CRP reach a strong SE $(87.2 \%)$ when performed together, even with a low SP $(25.9 \%)$. Furthermore, because the PCT and CRP combined assay was proven to be a statistically significant predictor of infection, its use would be of benefit to clinical routine practice. The other 3 predictors of infection that we found (age, presence of infection before ECMO implantation, and duration of ECMO support) cannot assist the medical attending team in day-to-day clinical decision-making. We showed that the PCT and CRP combined assay can aid in the diagnosis of infection in patients with VA ECMO, and further study will help us to analyze its role in the management of antibiotic therapy.

The low SP of the PCT and CRP combined assay is to be interpreted in light of the fact that we chose to privilege sensitivity to have a high NPV and to exclude the presence of infection as certain as possible if the test was negative. This observation may justify sparing antibiotic therapy for patients with a negative PCT and CRP combined assay test result, with a low error rate.

Our study also shows that PCT is not a reliable marker of infection in patients undergoing VV ECMO. Although the number of patients studied is small, we can argue that because the circuit is the same for VV ECMO and VA ECMO, such difference should be attributed to the patients. In particular, almost every patient with ARDS requiring VV ECMO had primary lung infection in contrast with the primary cardiac failure, which was typical of patients supported with VA ECMO. This observation paves the way for new studies to characterize the different categories of patients requiring ECMO treatment according to disease and comorbidity.

\section{Study Limitations}

The study has 4 important limitations. First, the small number of patients does not allow strong and general conclusions, even if the results are impressive. Second, distinguishing between SIRS and sepsis in patients on ECMO is an important problem. Nevertheless, these data would suggest no change in the clinical management of these patients, because the overlap among cardiogenic shock, 
SIRS, ECMO itself, and sepsis will always be part of the clinical scenario. Third, in a study conducted on 194 patients with community-acquired infection, Gaini and colleagues ${ }^{25}$ found that interleukin 6 , lipopolysaccharide binding protein, and CRP performed best in distinguishing between SIRS and sepsis, whereas PCT performed best in distinguishing between sepsis and severe sepsis. ${ }^{25}$ Because the patients included in the study had community-acquired infection instead of nosocomial infections and were not admitted to an ICU, it would be of interest to perform a similar study in the setting of critical care medicine. However, the transferability of this knowledge in the clinical application of ECMO is hard. Fourth, we were unable to identify a new test for the diagnosis of infection in patients undergoing $\mathrm{VV}$ ECMO, and further studies on this topic are strongly warranted.

\section{CONCLUSIONS}

We suggest a concomitant PCT and CRP assay with definite cutoff values as a new test to identify infection in patients undergoing VA ECMO. PCT routine dosing may be useful for infection diagnosis in patients requiring ECMO and would lead to a more rational and appropriate clinical management of these high-risk patients.

The authors thank Turla Otello Giancarlo and Motta Andrea, $\mathrm{MD}$, for the support in data collection and analysis.

\section{References}

1. Carlet J. Rapid diagnostic methods in the detection of sepsis. Infect Dis Clin North Am. 1999;13:483-94, xi.

2. Rivers E, Nguyen B, Havstad S, Ressler J, Muzzin A, Knoblich B, et al. Early goal-directed therapy in the treatment of severe sepsis and septic shock. $N$ Engl J Med. 2001;345:1368-77.

3. Dellinger RP, Levy MM, Carlet JM, Bion J, Parker MM, Jaeschke R, et al. Surviving Sepsis Campaign: international guidelines for management of severe sepsis and septic shock. Crit Care Med. 2008;36:296-327.

4. Horan TC, Andrus M, Dudeck MA. CDC/NHSN surveillance definition of health care-associated infection and criteria for specific types of infections in the acute care setting. Am J Infect Control. 2008;36:309-32.

5. Liang KY, Zeger SL. Regression analysis for correlated data. Annu Rev Pub Health. 1993;14:43-68.

6. Liang KY, Zeger SL. Longitudinal data analysis using generalized linear models. Biometrika. 1986;73:13-22.

7. Obuchowski NA. ROC analysis. AJR Am J Roentgenol. 2005;184:364-72.
8. DeLong ER, DeLong DM, Clarke-Pearson DL. Comparing the areas under two or more correlated receiver operating characteristic curves: a nonparametric approach. Biometrics. 1988;44:837-45.

9. Weinstein S, Obuchowski NA, Lieber ML. Clinical evaluation of diagnostic tests. AJR Am J Roentgenol. 2005;184:14-9.

10. Gardner IA, Stryhn H, Lind P, Collins MT. Conditional dependence between tests affects the diagnosis and surveillance of animal diseases. Prev Vet Med. 2000;45: 107-22.

11. Bizzarro MJ, Conrad SA, Kaufman DA, Rycus P. on behalf of the ELSO Task Force in Infections, ECMO. Infections acquired during extracorporeal membrane oxygenation in neonates, children, and adults. Pediatr Crit Care Med. 2011;12: 277-81.

12. Sun HY, Ko WJ, Tsai PR, Sun CC, Chang YY, Lee CW, et al. Infections occurring during extracorporeal membrane oxygenation use in adult patients. $J$ Thorac Cardiovasc Surg. 2010;140:1125-32.

13. Castelli GP, Pognani C, Meisner M, Stuani A, Bellomi D, Sgarbi L. Procalcitonin and C-reactive protein during systemic inflammatory response syndrome, sepsis and organ dysfunction. Crit Care. 2004;8:R234-42.

14. Arkader R, Troster EJ, Lopes MR, Jùnior RR, Carcillo JA, Leone C, et al. Procalcitonin does discriminate between sepsis and systemic inflammatory response syndrome. Arch Dis Child. 2006;91:117-20.

15. Prat C, Ricart P, Ruyra X, Domìniguez J, Morillas J, Blanco S, et al. Serum concentrations of procalcitonin after cardiac surgery. J Card Surg. 2008;23:627-32.

16. Sponholz C, Sakr Y, Reinhart K, Brunkhorst F. Diagnostic value and prognostic implications of serum procalcitonin after cardiac surgery: a systematic review of the literature. Crit Care. 2006;10:R145.

17. Gendrel D, Raymond J, Coste J, Moulin F, Lorrot M, Guèrin S, et al. Comparison of procalcitonin with C-reactive protein, interleukin 6 and interferon-alpha for differentiation of bacterial vs. viral infections. Pediatr Infect Dis. 1999;18: 875-81.

18. Marc E, Menager C, Moulin F, Stos B, Chalumeau M, Guèrin S, et al. Procalcitonin et meningitis virales: reduction des traitements antibiotiques inutiles par le dosage en routine au cours d'une epidemie. Arch Pediatr. 2002;9:358-64.

19. Shimetani N, Shimetani K, Mori M. Levels of three inflammation markers, C-reactive protein, serum amyloid A protein, and procalcitonin in the serum and cerebrospinal fluid of patients with meningitis. Scand J Clin Lab Invest. 2001;61:567-74.

20. Guervilly C, Coisel Y, Botelho-Nevers E, Dizier S, Castanier M, LepaulErcole R, et al. Significance of high levels of procalcitonin in patients with influenza A (H1N1) pneumonia. J Infect. 2010;61:355-8.

21. Eisenhut M. Use of procalcitonin measurement to identify bacterial co-infection in patients with H1N1 influenza. Acta Paediatr. 2010;99:487-8.

22. Meisner M, Tschaikowsky K, Hutzler A, Schick C, Schuttler J. Postoperative plasma concentrations of procalcitonin after different types of surgery. Intensive Care Med. 1998;24:680-4.

23. Hammer S, Fuchs AT, Rinker C, Daebritz S, Kozlik-Feldmann R, Netz H. Interleukin- 6 and procalcitonin in serum of children undergoing cardiac surgery with cardiopulmonary bypass. Acta Cardiol. 2004;59:624-9.

24. Picariello C, Lazzeri C, Chiostri M, Gensini GF, Valente S. Procalcitonin in patients with acute coronary syndrome and cardiogenic shock submitted to percutaneous coronary intervention. Intern Emerg Med. 2009;4:403-8.

25. Gaini S, Koldkjaer OG, Pedersen C, Pedersen SS. Procalcitonin, lipopolysaccharide-binding protein, interleukin-6 and C-reactive protein in community-acquired infections and sepsis: a prospective study. Crit Care. 2006;10:R53. 


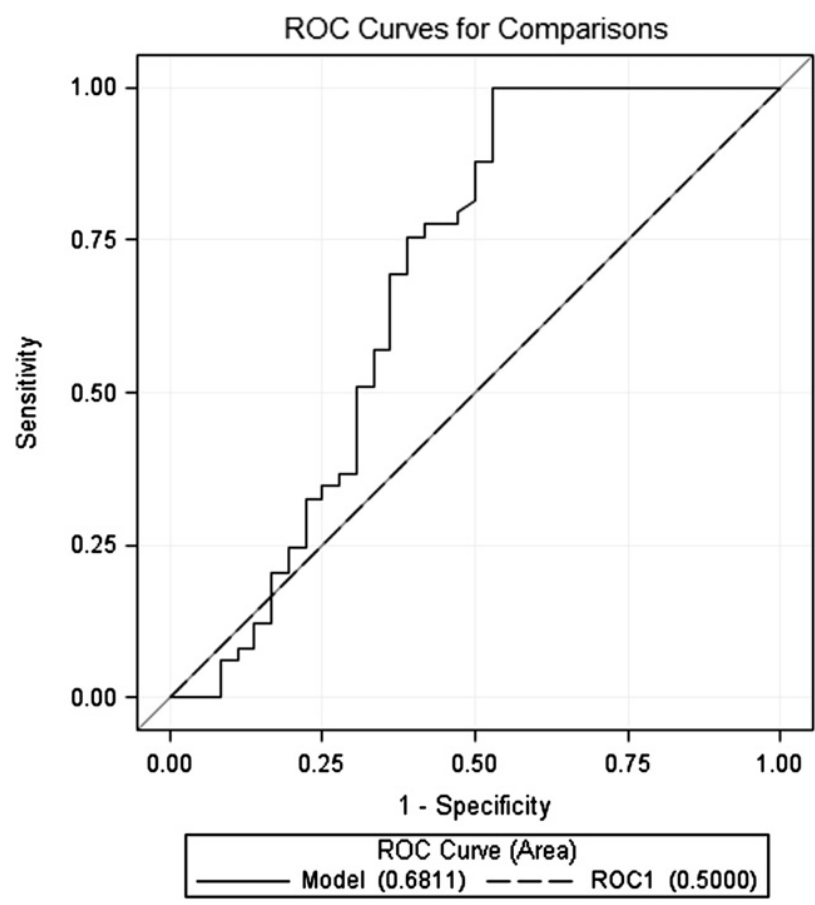

FIGURE E1. ROC curve of PCT in patients treated with VA ECMO. $R O C$, Receiver operating characteristic.

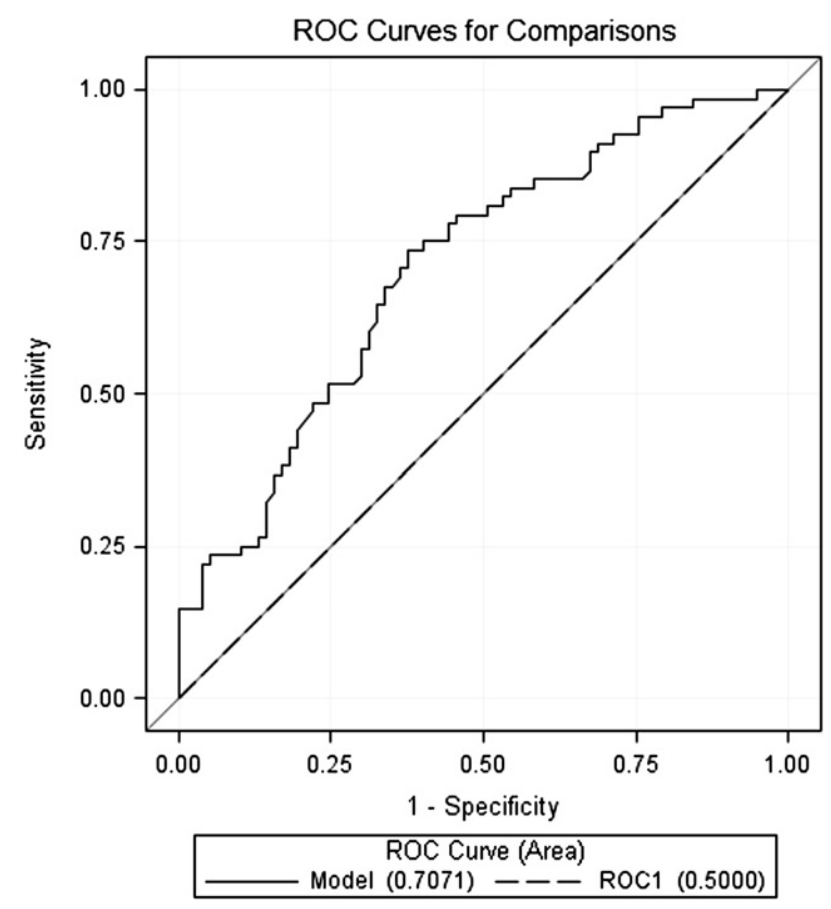

FIGURE E2. ROC curve of CRP in all patients treated with ECMO. ROC, Receiver operating characteristic. 